\title{
A COMPARATIVE REVIEW STUDY ON THE MANUFACTURING PROCESSES OF COMPOSITE GRID STRUCTURES
}

\author{
A. Fadavian, A. Davar*, J. E. Jam, S. H. Taghavian \\ Composite Research Center, Malek-e-Ashtar University of Technology, \\ Lavizan, Tehran, Iran
}

Received 28.01.2015

Accepted 06.04.2015

\begin{abstract}
Filament winding and fiber placement are low-cost, fast, and suitable processes for manufacturing composite grid structures. Resulted structures are high quality products. They have the advantage of carrying heavy structural loads as well as light structural weight. Composite Grid Structures (CGS) are manufactured with varying geometries such as circular (cylindrical and conic) and flat. They are applied in hightech industries including aerospace industry. In this paper, the manufacturing processes of these structures and their various aspects (including winding method, mandrel material and curing method) are reviewed and compared in detail.

Keywords: Composite Grid Structures, Filament Winding, Fiber Placement, Mandrel, Curing Cycle

\section{Introduction}

Today, the application of composite grid structures (CGS) is evident in many weight-sensitive areas in aerospace industry. These structures are applied to fairing, interstage, and adaptor structure of launch vehicle and fuselage of aircraft. This wide range of applications is due to their high specific strength and specific stiffness properties. In fact, these structures are substitutes for sandwich structures with honeycomb core and ring and stringer stiffened shells as well as aluminum grid structures. Grid structures' development goes back to the time when they were used in aircraft geodetic frames. Especially, they were applied to UK Wellington bombers in World War II. These bombers possessed metal geodetic frames. Yet, the history of
\end{abstract}

\footnotetext{
*Corresponding Author: Ali Davar, davar78@gmail.com
} 
Russian grid structures goes back to Moscow radio tower built in 1921. Shukhov designed it in $148.3 \mathrm{~m}$ height [1].

These CGS are manufactured using filament winding and fiber placement automatically. Huybrechts et al. [2,3] have surveyed these structures in America in a review paper regarding the works done on design, analysis and manufacturing methods. In another research, they have also examined mold manufacturing method in terms of hybrid tooling and expansion block. In a series of research works by Vasiliev et al. $[1,4,5]$, the history of composite grid structures manufactured in Moscow at the Central Research Institute for Special Machinery (CRISM), and design and analysis procedures are described based on continuum model using smearing the effect of the ribs to an orthotropic shell. Various manufacturing methods are also experienced, test methods, mechanical properties and aerospace applications of structures are mentioned. Totaro and De Nicola $[6,7,8]$ have described the results reported by Italian aerospace research center (CIRA) including designing, manufacturing and testing lattice cylinders produced by two methods: wet filament winding and dry winding. They compared results from structures tested under compressive axial load. Buragohain and Velmurugan $[9,10]$ built three composite cylinders (shell, lattice, and stiffened shell) using wet filament winding on foam mandrel. Then, they compared their specific buckling load by empirical and numerical methods. Chen et al. $[11,12,13]$ produced a sandwich structure with lattice core with two face sheets reinforced by two end stiff ring (flanges) using an innovative method. They compared failure modes under compressive axial load between this sample and samples manufactured without end flanges and internal skin (face sheet).

This paper reviews manufacturing methods of grid structures with various geometries including shells of revolution (e.g. circular cylinders and cones) and flat (panels). This study also examines and compares their different manufacturing aspects.

\section{Classifying a Variety of Grid Structures}

Composite grid structures are substitutes for aluminum grid structures, ring and stringer stiffened structures, and honeycomb sandwich structures. Yet, they are superior to the above structures in some aspects. Fig. 1. illustrates these structures.

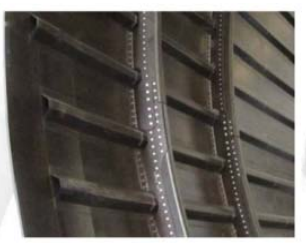

(a)

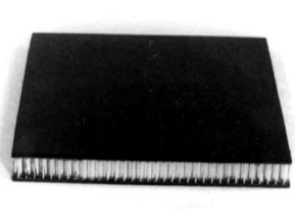

(b)

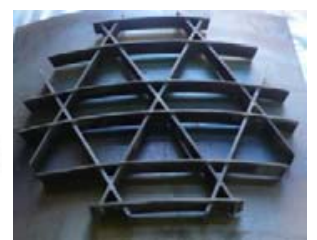

(c)

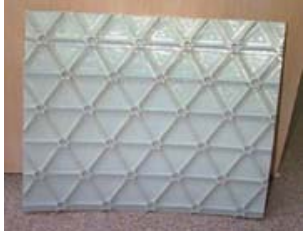

(d)

Fig. 1. Composite structures (a) stiffened with stringer and ring (b) sandwich structure with honeycomb core, (c) lattice structure, (d) aluminum lattice isogrid stiffened curved panel $[2,4]$.

These CGS are composed of an integrated grid by the placement of unidirectional composite ribs (tapes) in 2 to 4 directions (right-rounded and left-rounded cross, circumferential and longitudinal). Geometrically, they are classified into three groups: flat plates, open and closed shells of revolutions. These grids are generally without 
internal skin. This is because the main load-carrying components are ribs. Yet, they can have one or two skins based on the application environmental conditions [8]. Grid patterns are divided into two general groups: (1) isogrid demonstrating isotopic in-plane mechanical properties resulted from the organized and uniform orientations of the ribs; and (2) anisogrid demonstrating non-isotopic in-plane mechanical properties. Fig. 2. and 3. show some patterns.

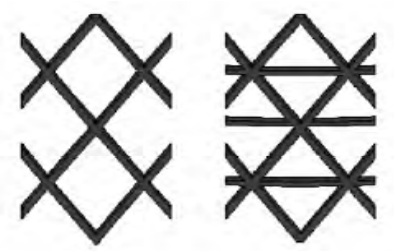

Fig. 2. Isogrid patterns [10]
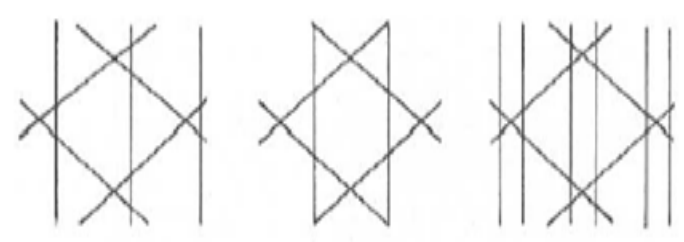

Fig. 3. Anisogrid patterns [1]

To create various configurations (patterns) in grid structures, designer has to consider parameters like number of ribs, cross section details, ribs interval, ribs angel, etc. Fig. 4. shows these parameters.

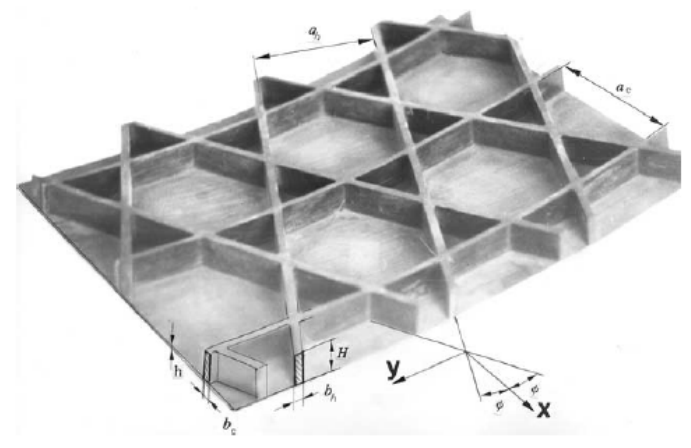

Fig. 4. Grid geometrical parameters [1]

Structures can be made of metal, composite, and a combination of them.

\section{The Advantages of Grid Structures versus Other Stiffened Structures}

Aluminum grid structures are produced by machining of thick aluminum plates. They have less stiffeness and more weight as compare to the composite structures. Based on the US Air Force Research Laboratory (AFRL) report, the composite sample of fairing was made and tested, $61 \%$ lighter, $300 \%$ stronger, and $1000 \%$ more stiffened as compared to its aluminum equivalent [2]. Both ring and stringer stiffened structures and sandwich structures with honeycomb are widely used in aerospace industry. They are based on the skin load carrying capability in these structures. The stiffeners in stiffened structures and the core in the sandwich structures provide high bending stiffness and resistance against buckling under compressive and shear loads. This is noteworthy that the stiffeners and the face sheets are not unidirectional in the ring and stringer stiffened and composite sandwich structures. But, they are laminated and 
including unidirectional composite layers with various orientation angels. Mechanical properties of these structures are considerably lower than the properties of their corresponding unidirectional composites of the same materials. For example, the inplane modulus of the quasi-isotropic structures $(0 / 90 /+45 /-45)$, widely used in the skin, are about $54 \mathrm{GPa}$. This is only $41.5 \%$ of the longitudinal modulus of the unidirectional carbon-epoxy composite (130GPa) and less than aluminum alloy modulus (70GPa) [4]. Load-carrying multilayered composite skins could not be connected to the adjacent metal or composite structural components easily. Usually, the bolted connections of load-bearing skins cannot transfer heavy loads due to the relatively low bearing strength of composite materials. Maximum authorized bearing stress does not exceed 160MPa for composites with epoxy matrix [4]. The excessive loads lead to residual strain. They are related to the microfracture of the material located near the bolt. As a result, composite structures stiffened by ring and stringer do not allow considerable weight reduction as compared aluminum aerospace structures. This is also true with sandwich structures. In contrast to the stiffened and sandwich structures, the strength and stiffness of grid composite structures are controlled by ribs. Both membrane and bending stiffness are provided by the ribs. It is important that ribs are manufactured by continuous filament winding or fiber placement and hence they have unidirectional structure. This leads to high specific strength and specific stiffness of the ribs. The advantages of grid structures (as compared to sandwich structures with honeycomb core) can be put in three parts [2].

1- Structural efficiency of these structures is higher. Due to the unidirectionality of the ribs. It is because the distribution of the applied loads at the direction of the ribs and maximum exploitation of the strength and the stiffness of the fibers in these directions increase CGS load-carrying properties.

2- The better performance of these structures in environmental conditions; CGS have higher damage tolerance. Grid structures have shown a tendency to contain delamination, a result of impact damage in a cell. They prevent from its spreading and catastrophic failure of the structure. Unlike honeycomb sandwich structures, these structures do not absorb and keep humidity during their service life. The absorbed humidity in honey comb core results in the corrosion of the structure.

3- Another advantage of the CGS is their automatic, continuous, and low-cost manufacturing process.

\section{The Manufacturing Processes of Composite Grid Structures}

Manufacturing of these types of composite structures can be classified from three viewpoints: 1- winding method (filament winding or fiber placement), 2- type of mold or mandrel, and 3- type of structure curing cycle. Production process is mainly aimed to provide appropriate quality and mechanical properties for (cross) spiral (for cone winding) or helical (for cylinder winding) ribs. These are the main load-carrying components in the structure. Winding is carried out in two ways: wet filament winding and dry (tape) winding. Wet filament winding is a process where a bundle of continuous fibers is dripped in resin pool is aligned on a circular mandrel by predetermined arrangement. When resin is cured, mandrel is separated from it and the process is finished. This method is usually applied to manufacturing circular grid structures (like cylinders and cones). Fig. 5. illustrates a schematic view of this process. 


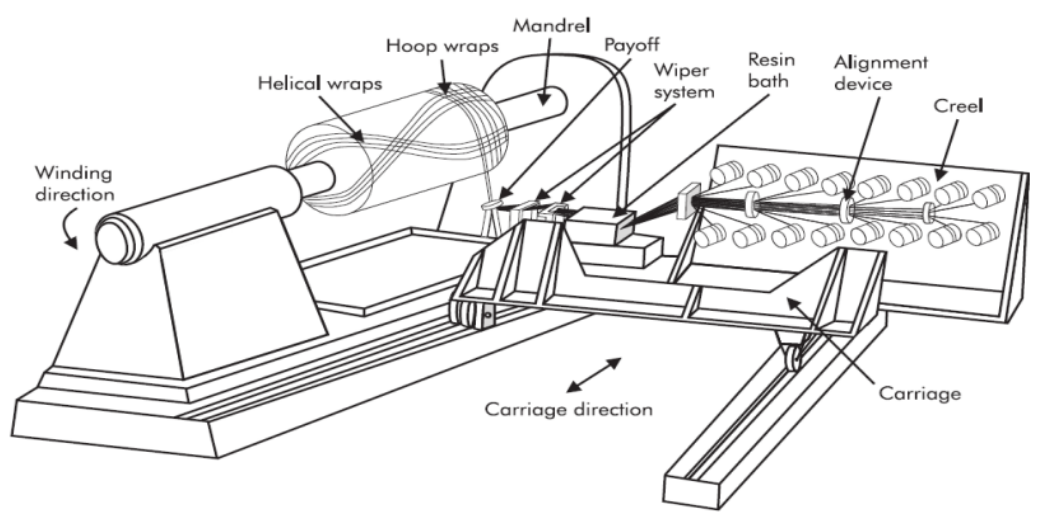

Fig. 5. A schematic view of wet filament winding process [14]

In dry winding process, prepreg tapes are in fact placed on desirable area by robot. This is also called fiber placement. Fig. 6 . shows this process.

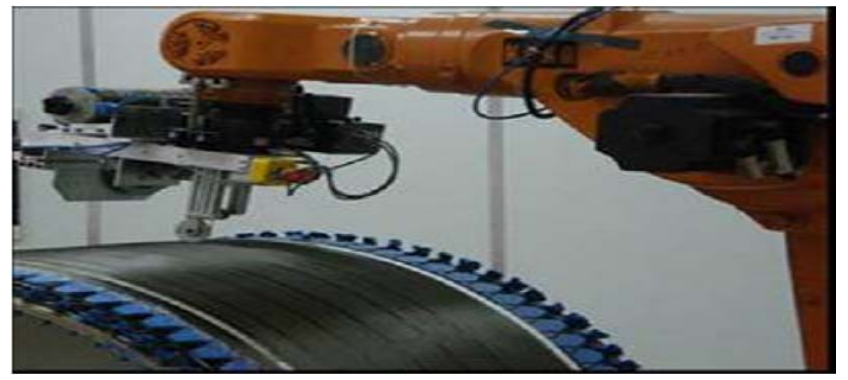

Fig. 6. Composite grid cylindrical dry winding process (fiber placement) [7]

These processes are generally done in two terms: conventional free winding and grooves (guided) winding. Unlike conventional free winding method where tapes cover total mandrel area composite tapes merely coat spiral or helical grooves. Then, external skin is obtained by circumferential winding. In this method, continuous winding of helical ribs is provided with the aid of radial pins placed at the end of the mandrel. Structures manufactured by this method are highly weight efficient. But, the external appearance quality of ribs is relatively poor. Fig. 7 . shows this mode.

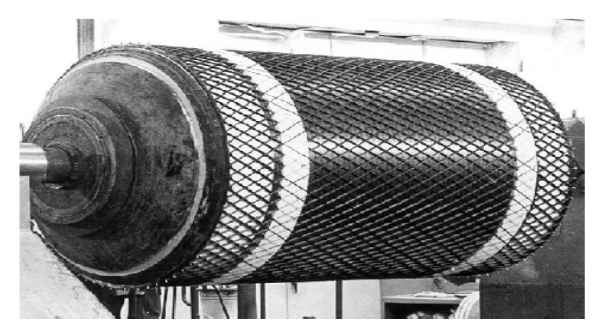

Fig. 7. Free filament winding [1] 
Grooves guided winding can be carried out on the mandrel composed of various materials. Metal mandrel (aluminum or steel) is made by helical and circumferential groove cutting. Mandrels must be made in several pieces. Then, they can be assembled on a shaft. They have suitable dimensional stability and are reusable (i.e. they can be used several times). At the same time, they are costly and time-consuming. Plaster mandrels are also made by molding plaster on a metal shaft.After creating grooves and winding, in order to extract the grid stiffened or lattice structure, plaster must be separated from the structure mechanically or via washing by water. Fig. 8. shows typical plaster mold.

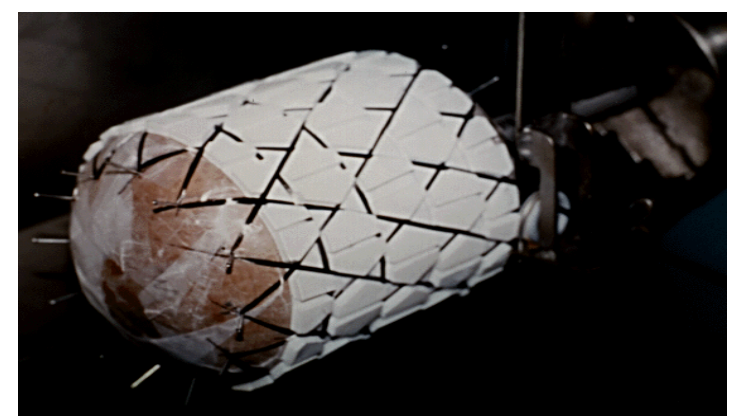

Fig. 8. A view of plaster mandrel [15]

Another type of mandrel is composed of polyurethane hard foam. For winding on core, light-weight hard foam usually includes wound internal skin. First, the sprayed core on the internal skin is machined and grooves are made by milling. Then, the ribs are wound inside the grooves in filament winding process. Finally, the external skin is overwrapped on the mandrel. This process is relatively costly. Relatively high mass of these structures enables them to carry heavy loads [1]. They have also good thermal insulation and vibration and acoustic damping properties. If these structures merely have external skin, foam can be separated from the structure (by mechanical, chemical or thermal decomposition). In foam mandrel, the detachment of a part of foam when cutting the grooves on the foam is an issue. Fig. 9. shows an example of these defects.

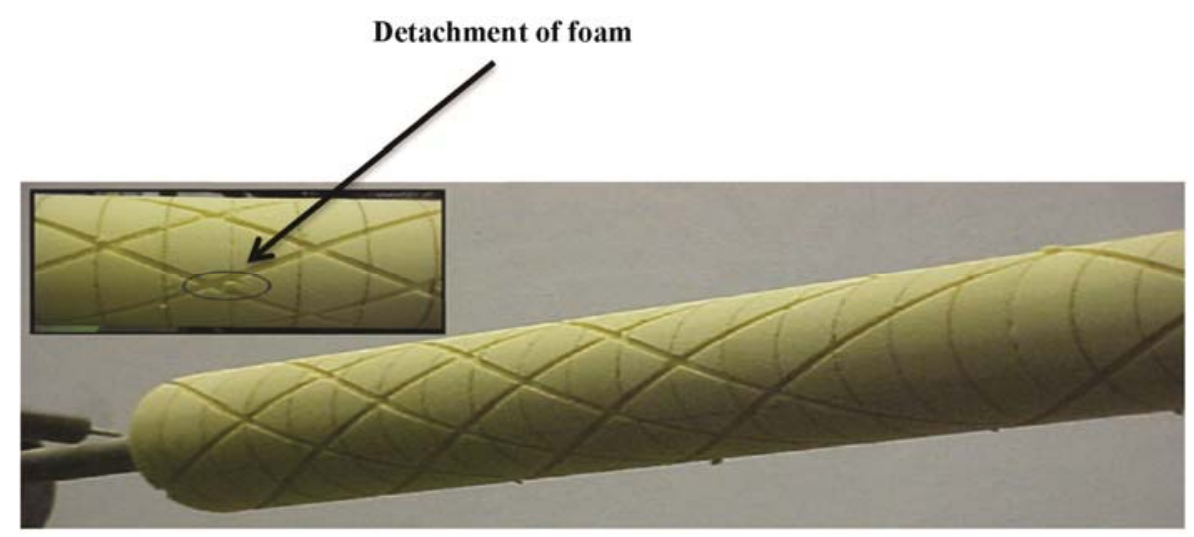

Fig. 9. Defections made in foam mandrel when machining crevices [9] 
The CGS with two skins is called sandwich structures with grid core. Recently, Chen et al. [9] have invented a method for manufacturing these structures. That is, grid structure is first wound on the metal mandrel. Then, external skin is wound. Vacuum bag molding is done and co-cured. Then, internal skin is wound on a metal mandrel, separately. It will be wedged in one end to be placed in previous structure which is reversely wedged. Then, both structures undergo vacuum bag molding and co-cured in autoclave. Fig. 10. shows a schematic view of this mold.

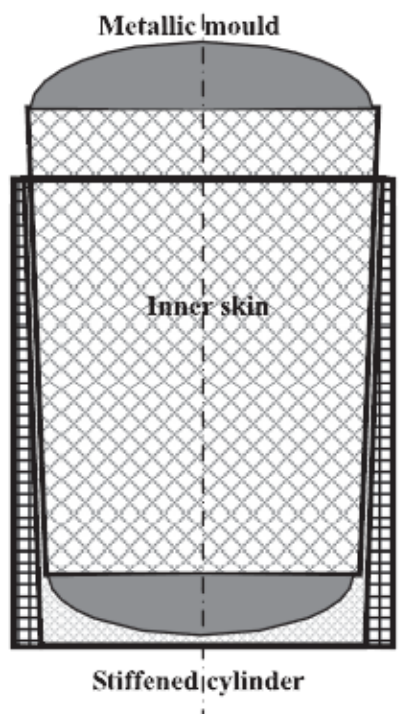

Fig. 10. Designing mandrel in wedge form to produce composite sandwich cylindrical structure with grid core [11]

Yet, thermoplastic mandrels like polyethylene and teflon are also used. Partially, all grid structures have the same manufacturing problems. In grid structures, multiple fiber bundles or prepreg tapes are passed through the ribs intersections. This causes the fibers to be composed and even prevents them to cross over together [3]. The aggregation of fibers in the ribs intersections causes the strength and stiffness to be decreased and accuracy in model to be increased. To remove this problem, two effective molding methods are used. Hybrid tooling and expansion block. In both methods lateral pressure is applied on the ribs during curing process. This is due to the thermal expansion coefficient of the materials used in these molds.

Hybrid tooling contains two main parts of different materials to form the structure. A base tooling (plate) and an expansion tooling (blocks). The plate is composed of stiff and thermally stable materials like epoxy. Grooves are machined in this part. Blocks are also made of materials with high thermal expansion coefficient like silicon rubber. It is cast in the grooves. Hence, the bundle of the fiber and prepreg tapes are placed in the grooves. The expansion part laterally compresses ribs during curing. As a result, air bubbles (voids) exit from the resin and composite quality is increased. Fig. 11. shows examples of this mold. 

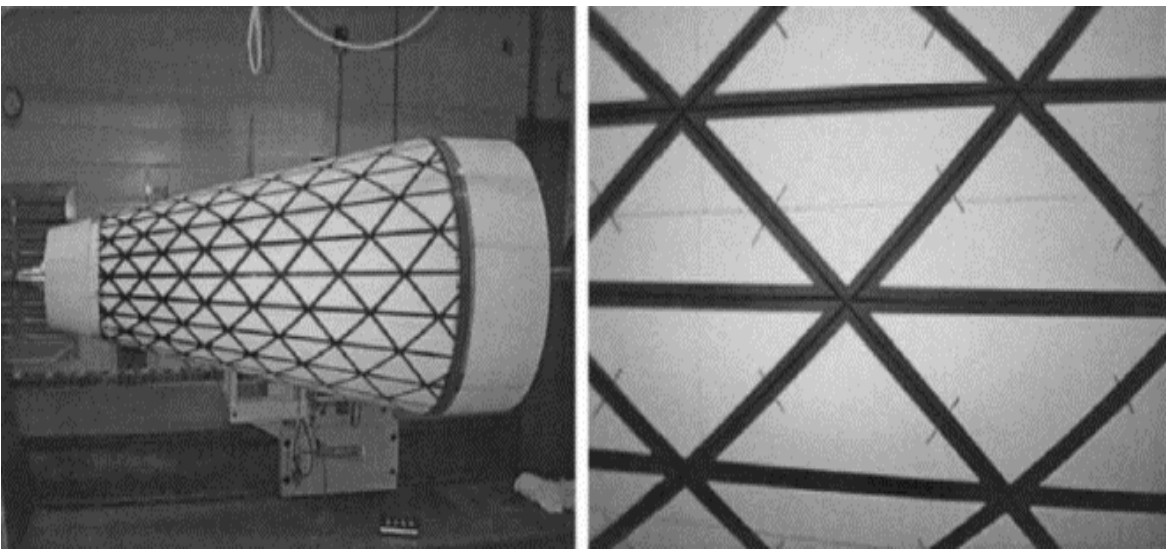

Fig. 11. An example of hybrid tooling where the basic part is made of epoxy and expansion part is made of silicon rubber [3]

The expansion mold is very similar to hybrid tooling, but the expansion blocks are incompressible materials with high thermal expansion coefficient. They are bolted to the base plate. Fig. 12. shows a model of this tooling.

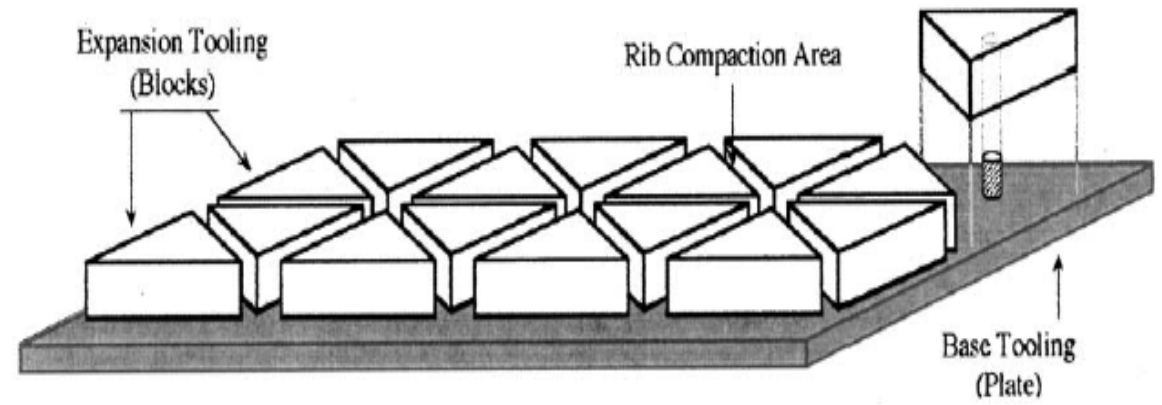

Fig. 12. A model of expansion tooling [3]

Two previous methods are mostly used for producing flat grid structures [3]. Yet, silicon carpet is the most recent invented method. The silicon carpet can be produced in two ways:

1- Casting the liquid silicon in the grid structure male mold, and 2-Creating the grooves by special tool in silicon sheet. Fig. 13. shows an example of molded silicon carpet. 


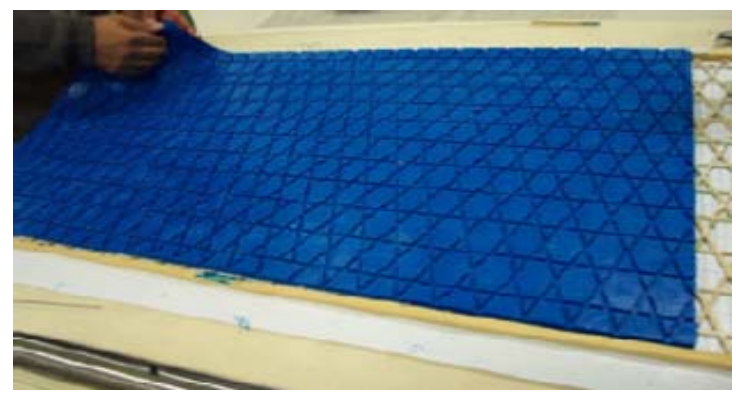

Fig. 13. Silicon carpet made by molding method [6]

Fig. 14. shows different stages of composite cylindrical grid structure manufacturing process.

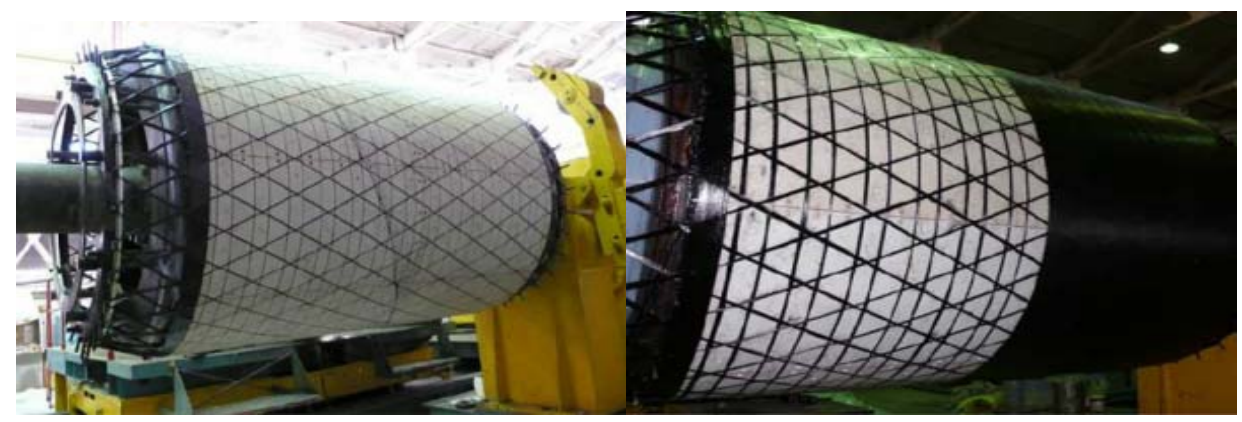

(a)

(b)

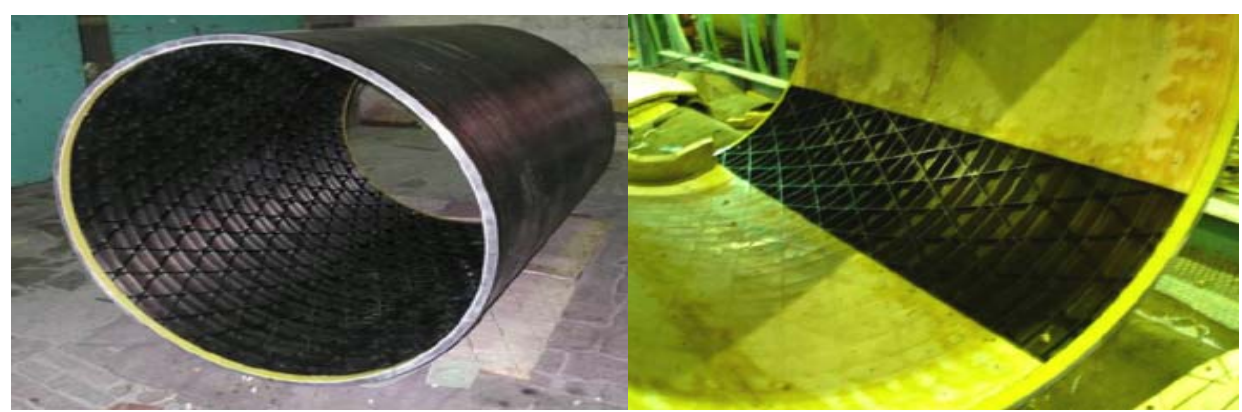

(c)

(d)

Fig. 14. (a) ribs winding, (b) skin winding (c) separating rubber coating, (d) manufactured structure [4]

Resins used in these structures are usually hot cured. As a result, they are cured in autoclave or oven after vacuum bag molding.

\section{Conclusions}

Different methods of manufacturing flat and curved composite grid stiffened structures were studied and compared. 
It is to be noted that grid structures manufactured by prepreg tapes are of less quality as compared to wet processes. It is mainly due to low resin content placed in the grooves. In fact, the resin percentage (resin volume fraction) is predetermined in prepreg tapes and it cannot be changed. The invariable resin percentage increases fiber volume fraction at the intersection of the ribs (nodes). This increase results in the heterogeneity and susceptibility to crack initiation at these points. The useful outcomes of the present study as follows and can be classified into three parts:

1- It is possible to control resin percentage to fill the ribs using wet filament winding. Low resin viscosity (as compared to prepreg tapes) produces suitable wetting of the fibers in the resin matrix. In this method, the ribs are homogenous and relatively well organized. It will considerably increase the structure load-carrying capability as compared to the same structure made by dry winding.

2- For manufacturing flat or curved CGS, silicon rubber coating is superior to other materials used in manufacturing the molds or mandrels. This is because the silicon rubber applies lateral pressure on the ribs during curing process. Resin bubbles exit as a result of this lateral pressure and consequently composite quality is increased.

3- Curing method depends on the method of manufacturing in winding, i.e. filament winding or fiber placement. Usually, in fiber placement, the structure is cured in autoclave when prepreg tapes are placed and vacuum bag molding is applied to obtain higher quality. But, in wet filament winding, the structure can be cured in the oven after being wound and vacuum bag molded. This reduces production costs considerably as compared to the fiber placement method.

Generally, selecting the manufacturing method of composite components and structures depends on two main factors: quality and cost.

\section{References}

[1] V. V. Vasiliev, V. A. Barynin, A. F. Rasin, Compos. Struct. 54 (2001) 361-370.

[2] S. M. Huybrechts, S. E. Hann, T. E. Meink, In: Proceedings of the $12^{\text {th }}$ International Conference on Composite Materials (ICCM12). Paris, France, 1999.

[3] S. M. Huybrechts, T. E. Meink, P. M. Wegner, J. M. Ganley, Composites Part A. 33 (2002) $155-161$.

[4] V. V. Vasiliev, V. A. Barynin, A. F. Rasin, Compos. Struct. 94 (2012) 182-189.

[5] V. V. Vasiliev, A. F. Rasin, Compos. Struct. 76 (2006) 1117-1127.

[6] G. Totaro, F. De Nicola, In: Proceedings of the AIAA/CIRA 13th International Space Planes and Hypersonic Systems, Capua, Italy, 2005.

[7] G. Totaro, F. De Nicola, Acta Astronaut. 81 (2012) 570-577.

[8] G. Totaro, PhD Thesis, Delft University of Technology, Delft, South Holland, Netherlands.See also URL http://www.tudelft.nl/en 2011.

[9] M. Buragohain, R. Velmurugan, Compos. Struct. 93 (2011) 1031-1038.

[10] M. Buragohain, R. Velmurugan, Def. Sci. J. 61 (2011) 88-94.

[11] L. Chen, H, Fan, F. Sun, L. Zhao, D. Fang, Thin Walled Struct. 68 (2013) 75-84.

[12] T. Kim, Compos. Struct. 45 (1999) 1-6.

[13] H. Fan, D. Fang, L. Chen, Z. Dai, W. Yang, Compos. Sci. Technol. 69 (2009) 2695-2700.

[14] A. B. Strong, Fundamentals of composites manufacturing (materials, methods and applications), second ed., SME, Michigan, 2008.

[15] A. Hou, K. Gramoll, J. Aerosp. Eng. 12 (1999) 83-91. 